\title{
Infraestructura y movilidad: Una reflexión histórica comparativa sobre Chile y México, 1840-19801
}

\author{
Guillermo Guajardo Soto² \\ Recibido: 10 de febrero de 2014 Aprobado: 25 de marzo de 2014
}

\begin{abstract}
Resumen
Estaciones ferroviarias, posadas, terminales de autobuses y aeropuertos han sido el soporte material que por más de un siglo se han empleado para mover personas y bienes, pero también para brindar el descanso y la conexión hacia nuevas rutas y otros medios de transporte. Desde el siglo XIX infraestructuras e instalaciones se construyeron para establecer los flujos de larga distancia hacia los mercados de trabajo, la guerra y más tarde del turismo. En particular analizamos las dinámicas relaciones entre la construcción y operación de la infraestructura y de los modos de transporte para producir la movilidad en Chile y México entre los decenios de 1840 y 1980.
\end{abstract}

Palabras clave: Infraestructura, Movilidad, Territorio, Transporte.

\section{Infrastructure and Mobility: A Comparative Historical Reflection on Chile and Mexico, 1840-1980}

\begin{abstract}
Railway stations, lodges, bus terminals and airports have been the material support that for more than a century have been used to move people and goods, but also to provide rest and connection to new routes and other means of transport. From the nineteenth century were built infrastructure and facilities to establish long distance flows to labor markets, war and, later, tourism. In
\end{abstract}

1 Este artículo recoge algunas de las líneas de análisis del proyecto principal del autor titulado "Tecnologías, organizaciones e infraestructuras para la innovación y la movilidad: un enfoque histórico interdisciplinario" que desarrolla en el Centro de Investigaciones Interdisciplinarias en Ciencias y Humanidades, $\mathrm{CE} \| \mathrm{CH}$, de la Universidad Nacional Autónoma de México, UNAM. Una primera versión fue presentada y discutida como ponencia en el Coloquio "Étapes, escales et relais en Amérique latine: cinq siècles d'histoire des haltes" en la Universidad Michel de Montaigne Bordeaux 3, Burdeos, Francia, octubre de 2013.

2 Chileno-mexicano, Doctor en Estudios Latinoamericanos, Universidad Nacional Autónoma de México (UNAM). Investigador Titular en el Centro de Investigaciones Interdisciplinarias en Ciencias y Humanidades (CEIICH) de la UNAM e Investigador del Sistema Nacional de Investigadores-Conacyt México. E-mail: guillermo.guajardo@unam.mx 
particular, we analyze the dynamic relationships between the construction and operation of infrastructure and transport modes to produce mobility in Chile and Mexico between the 1840s and 1980s.

Key words: Infrastructure, Mobility, Territory, Transportation.

\title{
Infraestrutura e mobilidade: Uma reflexão histórica comparativa sobre Chile e México, 1840-1980
}

\begin{abstract}
Resumem
Estações ferroviárias, posadas, terminais de ônibus e aeroportos tem sido o suporte material que por mais de um século tem-se utilizado para mover pessoas e bens, porém também para brindar o descanso e a conexão para novas rotas e outros meios de transporte. Desde o século XIX infraestruturas e instalações se constroem para estabelecer os fluxos de longa distancia para os mercados de trabalho, a guerra e mais tarde do turismo. Em particular analisamos as dinâmicas relações entre a construção e operação da infraestrutura e dos modos de transporte para produzir a mobilidade no Chile e México entre os decênios de 1840 y 1980.
\end{abstract}

Palavras chave: Infraestrutura, Mobilidade, Território, Transporte.

\section{La infraestructura y las instalaciones fijas para los flujos}

Los desplazamientos de bienes y personas han dejado una arqueología de soportes materiales, equipos, así como una historia de rutinas, procesos y conocimientos sobre cómo iniciar y concluir un viaje, constituyéndose las infraestructuras fijas -como las estaciones ferroviarias y los aeropuertos- en el testimonio físico de los flujos, el soporte material del movimiento humano (Prelorenzo, 2007: 85). Fue desde la Revolución Industrial cuando empezaron a expandirse grandes sistemas socio-técnicos que establecieron una coordinación global de transportes e información, la cual fue gestionada a través de ferrocarriles, telégrafos y puertos. Ello se amplió durante el llamado período fordista con la carretera, el camión y el avión, y tras el fin de la Guerra Fría se registra un intenso movimiento global de factores e información, acompañado de actividades rutinarias y regulares que son básicas para la vida humana y que, sin embargo, no han recibido una atención suficiente por parte de la historia (Edgerton, 2007; Van der Vleuten, 2008: 974, 978).

La construcción de este sistema más integrado de infraestructuras, flujos, etapas y puntos logísticos, queremos analizarlo comparativamente en los casos de Chile y México entre los decenios de 1840 y 1980, dos casos ubicados en los polos de la región latinoamericana y con diferenciados grados 
de acceso a la economía global. En particular nos interesa la "producción" de la movilidad de larga distancia para el trabajo, la guerra y el descanso.

Esta aproximación plantea el impacto relativo que tuvieron los nuevos medios mecanizados de transporte en su cobertura territorial, demográfica y en las velocidades, destacándose que se mantuvieron las largas y lentas jornadas de desplazamiento en amplias regiones latinoamericanas. Los antiguos caminos coloniales fueron la subestructura para los nuevos medios, perpetuándose la vieja orientación de las rutas y retrasándose el acceso a los polos económicos más dinámicos. Desde el siglo XIX los nuevos medios de transportes e infraestructuras introdujeron una mayor velocidad en la movilidad de bienes y personas, pero su impacto fue relativo por la limitada cobertura territorial de la infraestructura de vías férreas y caminos, prolongándose las velocidades bajas y un gran número de etapas de descanso y de reposta en las rutas terrestres en el siglo XX, situación que empezó a romperse con los aeropuertos, pero para un pequeño segmento de la población. Nuestro punto de vista es escéptico sobre las grandes narrativas que han enfatizado en el impacto radical y revolucionario de los nuevos medios de transporte (que en algún momento el autor también compartió), proponiendo un panorama más variado sobre los tiempos y las velocidades de movilidad de orden económico y social.

\section{Chile: la economía campesina del desplazamiento}

Un primer caso es el de Chile, país que desde el siglo XIX moldeó su sociedad y economía a través de los ciclos exportadores de materias primas, predominantemente mineras: plata (1830-1860), primer ciclo del cobre (18301880), nitrato (1880-1930) y segundo ciclo del cobre (1915-1970). Aunque en lo político y social fue un país agrario, dominado por los hacendados que definieron hacia mediados del siglo XIX una división del trabajo entre el Estado, que asumió la construcción de ferrocarriles en la zona central y sur del país en donde estaban los poderes públicos, gran parte de la población y una agricultura comercial. El capital privado, en tanto, se encargó de construir sus ferrocarriles, caminos y puertos para cubrir estrictamente las necesidades de exportación de minerales, generalmente en regiones poco habitadas y desérticas del norte del país. Esta división construyó dos sub-países, el primero estatal y con un mercado laboral más integrado en torno al campo y luego a la ciudad; el segundo se caracterizó por estar muy mal conectado con el centro y especializado en los desplazamientos de bienes mineros.

Así, en el primer sub-país estructurado desde el núcleo entre el puerto de Valparaíso, la capital Santiago y la ciudad de Concepción, después de las guerras de Independencia (1810-1818) la presión productiva y demográfica llevó en 1842 a crear el Cuerpo de Ingenieros Civiles para mantener y construir caminos en la zona central y agrícola, sin embargo, los costos de reparar eran 
altos y los grandes usuarios, como los hacendados, no cooperaban porque consideraban que era una tarea del poder público. Para ese entonces gran parte del transporte terrestre era brindado por una economía campesina de operadores, avituallamiento, comidas y refugio para las largas jornadas de carretas arrastradas por bueyes. A inicios de la década de 1840 circulaban alrededor de ochocientos de esos vehículos entre Santiago y el puerto de Valparaíso, cuyos dueños eran "chacareros" e "inquilinos" de las haciendas vecinas, quienes conformaban un empresariado popular del transporte. En la década de 1860 el viaje ida y vuelta entre la capital, Santiago, y el puerto de Valparaíso, de aproximadamente ciento treinta kilómetros, se hacía en catorce o quince días. En el camino había posadas, pero los carreteros por su pobreza comían al aire libre y dormían sobre cueros y pieles de vaca (Cáceres Muñoz, 2004: 61-70; Gay, 1973: 222-247).

\section{Ferrocarriles vs. caminos y la continuidad de las velocidades pre-industriales}

El panorama anterior empezó a cambiar desde la década de 1850 con la construcción de vías férreas para unir los puertos con los yacimientos mineros y también con las principales ciudades del interior del país. Este medio de transporte vino a solucionar el principal obstáculo para la intensificación de los cultivos y brindó una disminución tanto en el costo de los fletes como en los tiempos del viaje que se redujeron de días a horas, a la vez que se valorizaron los terrenos cercanos a la vía. En el caso de la ruta Santiago a Valparaíso se optó por trazar una ruta más al norte del camino de carretas, de alrededor de 187 kilómetros que quedó terminada a mediados del decenio de 1860 y que redujo el viaje a un par de horas en el día (Guajardo 2007: 9).

El otro lado de la moneda fue que aceleró la destrucción de buena parte de la economía campesina del transporte, a la vez que se descuidó la construcción y mantenimiento de los caminos. Por ello, a inicios del siglo XX había fuertes presiones de diversos sectores productivos para que el Estado superara las pésimas condiciones de la red caminera, especialmente en la zona central y sur en donde las lluvias de invierno los hacían intransitables.

En el segundo sub-país, en el norte minero, los caminos estaban menos desarrollados y eran difíciles por las condiciones del desierto, la escasez de animales de tiro y de fuerza de trabajo. Para solucionar esta situación, en 1908 el gobierno chileno empezó a avanzar desde el sub-país estatal con la construcción del Ferrocarril Longitudinal Norte (FCLN). Sin embargo, volvió a marcarse la división del pacto oligárquico entre el Estado y el sector privado, ya que en la zona central y sur del país se uniformó la vía a un ancho de 1,676 mt., en cambio en el FCLN se redujo a un metro y se mantuvieron varios tipos 
de anchos, como también se ahorró en la construcción de túneles y las pronunciadas cuestas obligaron a mantener trasbordos de pasajeros y mercaderías.

En 1913, por ejemplo, el viaje entre la capital, Santiago, y La Serena, en el norte del país, de 618 kilómetros por ferrocarril (hoy de 478 kilómetros por carretera) era descrito como muy deficiente, según lo anotaba el ingeniero Santiago Marín Vicuña (Marín Vicuña, 1914: 6-7); el viaje entre ambas ciudades era de tres días con locomotoras de vapor, cumpliendo diez etapas entre terminales, estaciones, trasbordo a otro tren y de alojamientos para comida y descanso, con una velocidad promedio de entre 17 a 23 kilómetros por hora, que se desglosaban de la siguiente manera:

DÍA 1

$8 \mathrm{hrs} . \quad$ Salida de Santiago, en tren de 1,676 de ancho;

10:30 hrs. Trasbordo en Calera, paso a la vía de un metro de ancho;

13 hrs. Trasbordo en Cabildo;

20 hrs. Trasbordo en Limáhuida;

21:30 hrs. Se llega "rendido y famélico" a un primer alojamiento en Illapel para comer y dormir.

DÍA 2

8:30 hrs. Salida de llapel;

15 hrs. Trasbordo en San Marcos;

18:30 hrs. Llegada a Ovalle a un segundo alojamiento para comer y dormir

DÍA 3

10:30 hrs. Salida de Ovalle;

16:30 hrs. Llegada a La Serena.

El viaje ferroviario en 1913 hacia el norte del país, región exportadora de la cual dependían las finanzas públicas, demoraba tanto como un viaje por carreta en el siglo XIX, siendo más rápido hacerlo en barco. Por lo demás el "vía crucis" para los pasajeros también lo era para la carga, ya que por falta de acuerdo entre las compañías no era posible trasladar minerales. Todo esto era producto del "pacto de caballeros" entre el país minero y el país agrario, subordinándose la técnica para impedir que la intervención pública entrara al dominio de las líneas privadas que recogían los fletes más lucrativos de la exportación minera. Esa decisión fue duradera, ya que para la década de 1960 si bien se introdujo la tracción diesel y mejoró la geometría de la vía, el viaje seguía siendo más lento con respecto a los viajes de la zona central y sur: en 1966 el viaje de tres días se había reducido a 27 o 28 horas (Ferrocarriles del Estado, 1966).

La situación anterior también definió la movilidad de los conflictos militares. Chile en el siglo XIX participó en varias guerras internacionales cuyo desenlace fue marítimo, en las que el ferrocarril no tuvo un papel relevante en la táctica y 
estrategia militar. Este medio de transporte más bien fue empleado para movilizar tropas desde el centro del país, desde donde eran despachados en barcos a los teatros de operaciones durante la guerra contra Perú y Bolivia (1879-1883).

Posteriormente las sucesivas lecciones militares alejaron a las fuerzas armadas chilenas del ferrocarril por varias razones. La primera fue que a diferencia de la guerra civil en los Estados Unidos y de la guerra Franco-Prusiana, en donde los ferrocarriles acercaron a las tropas al frente de guerra o a las fronteras, en Chile la barrera de la cordillera de los Andes y la lejanía de los desiertos del norte definieron escenarios de guerra internacional con participación de medios navales. Una segunda razón fue que la huelga ferroviaria de 1907 paralizó el centro y sur del país, anulando cualquier capacidad de desplazar tropas. Las lecciones de la Primera Guerra Mundial indicaron que una posible guerra en Chile tendría por escenario zonas de montaña y el mar. Finalmente por motivos políticos, los militares en las décadas de 1920 y 1930 no pudieron quitarle el control de los ferrocarriles estatales a la tecnocracia de los ingenieros civiles (Guajardo, 2008).

\section{México: la activación de los desplazamientos de mano de obra}

En el caso de México los viejos troncales de caminos coloniales indicaron las rutas para los ferrocarriles que avanzaron muy lentamente; la primera concesión se otorgó en 1837 y la operación completa de la vía entre la Ciudad de México y el puerto de Veracruz tuvo lugar en 1873. Las líneas se construyeron sobre una matriz de circulación interna que se había establecido durante el dominio colonial que ubicó a la Ciudad de México como el centro de una estrella. El plan de vías férreas trazado durante la ocupación francesa del país entre 1863 y 1867, pretendía complementar esa estructura con vías y caminos transversales sin privilegiar la conexión con los Estados Unidos. Pero tras la restauración republicana ello cambió con la construcción entre 1881 y 1884 de más de 4.500 kilómetros de vías que, en gran parte, comunicaron el centro con la frontera norte de México (Chapman, 1975; Guajardo 2010a: 183-190).

Las vías férreas hicieron posible una nueva movilidad para los productores rurales en ferias comerciales, exposiciones agroganaderas, la circulación de agentes viajeros así como la activación comercial de diversos actores sociales, como las mujeres zapotecas del Istmo de Tehuantepec que se convirtieron en agentes viajeras, consolidando así nuevos circuitos comerciales y redes sociales (Van Hoy, 2008). El medio de transporte creó nuevos flujos de fuerza de trabajo del centro hacia el norte del país, como consecuencia de despojos agrarios y por el atractivo de los salarios, relativamente más altos. Además los gobiernos de los generales Manuel González (1880-1884) y Porfirio Díaz (1884-1911) 
otorgaron facilidades a las compañías constructoras para reclutar y movilizar mano de obra, lo que significó una rápida proletarización que se inició con los peones de la vía y continuó en los talleres de reparación (Guajardo, 2010b: 52).

\section{Etapas y puntos logísticos de la cultura del trabajo}

El nuevo medio de transporte creó un flujo laboral hacia los Estados Unidos para el mantenimiento de las vías y las cosechas de algodón en Texas, Louisiana y en otros estados del sur estadounidense, pero también gestó un flujo de trabajadores hacia México desde el Golfo de México, las Antillas y el Caribe para la construcción de vías férreas. En la década de 1880 se emplearon alrededor de veinticinco mil trabajadores, de los cuales alrededor de diez mil pasaron a trabajar en la operación cotidiana de los ferrocarriles (Leal y Woldenberg, 1986: 96-97).Esto también estimuló la llegada de anglosajones que llegaron como maquinistas y mecánicos; hacia 1890 cerca de dos mil ciudadanos estadounidenses residían en Chihuahua, muchos de los cuales eran empleados ferrocarrileros. También había grandes colonias de ferroviarios estadounidenses en Monterrey, San Luis Potosí, Aguascalientes, Torreón y en la Ciudad de México, que contribuyeron a la americanización de sus barrios. Hacia 1910 los estadounidenses eran el tercer grupo de extranjeros en México, con 20.639 personas, dentro de un total de 117.119 extranjeros (Guajardo, 2010B: 53-54).

Todo lo anterior para 1910 conformó ejes y etapas ferroviarias para los flujos laborales; en el centro y sureste del país destacaba el eje del puerto de Veracruz a la ciudad de México, con puntos de proletarización, asociados a talleres de mantenimiento como Puebla y Orizaba en donde crecían barrios obreros importantes. También hubo un nuevo flujo, el de las ideas difundido por la Iglesia metodista episcopal con sede en Nueva York, cuya doctrina asociaba el progreso material para crear una nueva mentalidad popular ascética. Tras la terminación del Ferrocarril Mexicano llegó el primer pastor, que desde 1873 instaló templos y escuelas siguiendo el eje ferroviario de Veracruz, Puebla y la Ciudad de México para luego ubicarse en ciudades que contaban con ferrocarril e industrias (Guajardo, 2010b: 46; Ruiz, 1992).

\section{Revolución, caminos y automotores}

Un fenómeno relevante y definitivo en los desplazamientos mexicanos se dio durante la Revolución Mexicana que entre 1910 y 1920 transformó al ferrocarril desde una función económica a una militar para movilizar amplias masas campesinas hacia las zonas en donde se requería su presencia militar. El mayor aprovechamiento estuvo por el lado de los rebeldes, quienes adaptaron la logística comercial y distributiva de los trenes para enfrentar a las fuerzas del gobierno, que carecían de un enfoque moderno de movilidad, refugiándose 
en las guarniciones estáticas y la caballería. La Revolución Mexicana también abrió el paso al autotransporte, primero en las ciudades, y posteriormente en algunos combates en donde el uso de automóviles y camiones reemplazó a las caballerías, abriendo paso al poder político de los transportistas en un país con una escasa cobertura ferroviaria.

La logística ferrocarrilera, que implicó poco más de dos mil estaciones para 1910 (Guajardo y Riguzzi, 2013), si bien rompió los moldes tradicionales su influjo operó en ejes muy específicos, debido a que por la débil cobertura de los caminos se retrasó su impacto. Para principios de la década de 1930 el 93\% de los pueblos rurales no tenía acceso al ferrocarril, mientras que el $95 \%$ no contaba con telégrafos (Wilkie, 1987: 249,250). Un mayor efecto lo tuvo el autotransporte después de la Revolución gracias a una política de caminos desarrollada a contar de 1925, aunque en ese entonces los conductores de camiones debían abrir brechas con pala y maquinarias para establecer su negocio. En la década de 1930 camiones y autobuses irían detrás de las excavadoras que construían las carreteras. Un nuevo ciclo de movimiento empezó con el autobús que facilitó la migración del campo a la ciudad, en tanto que el camión abrió mercados más amplios para el campesino (Guajardo, 2010a).

\section{Viajes y descanso en el siglo XX}

El autotransporte se presentó como más flexible que el ferrocarril, pero dependió de la orientación y avituallamiento de gasolina, descanso y mantenimiento de las ciudades y de los caminos que, en general, privilegiaban la conexión con las capitales de los estados, por lo que un viaje hacia la capital del país, el Distrito Federal/Ciudad de México debía seguir antiguas rutas con muchas etapas de descanso, reposta y alimentación, incluso hasta la década de 1980. Según testimonios, en el decenio de 1970 el viaje en autobús a la Ciudad de México desde pueblos de la región del Istmo de Tehuantepec, en el estado de Oaxaca, cercanos al Océano Pacífico podía demorar de 30 a 34 horas, lo que actualmente se ha reducido a 12 horas porque las nuevas carreteras toman trazos directos. Desde el Istmo se debía pasar por la capital del estado, la ciudad de Oaxaca, en autobuses que desde las regiones rurales llevaban tanto personas, como bultos y animales de corral; ${ }^{3}$ tal como en Chile en la década de 1840, en el México del decenio de 1970 los viajeros y conductores recurrían a la economía campesina a la vera del camino que ofrecía agua de coco, cervezas, empanadas de plátano, tostadas de maíz y guisos diversos.

3 Entrevista al señor Emilio Escobar Velázquez, en el pueblo de Santo Domingo Zanatepec, región del Istmo de Tehuantepec, estado de Oaxaca, México, 20 de diciembre de 2012. 
Caminos y autobuses intensificaron los flujos de trabajo pero también hicieron posible el descanso masivo, siendo Acapulco sobre la costa del Pacífico el ejemplo más conocido desde el decenio de 1930, que se desarrolló gracias a las empresas de autobuses y más tarde de aviación. Desde el decenio de 1970 nuevos destinos turísticos quedaron a cargo casi exclusivamente de la aviación, en especial porque se establecieron vuelos directos desde los Estados Unidos sin hacer escala en el aeropuerto de la Ciudad de México; esto último favoreció al polo turístico de Cancún, ubicado en el Caribe mexicano basado en la disponibilidad de comunicación aérea con los Estados Unidos (Macías y Pérez, 2009; Boggio, 2008; Torres, 2003).

Cancún fue un proyecto del gobierno mexicano para atraer inversiones en hoteles, construyendo el sector público toda la infraestructura para servicios básicos de energía y comunicaciones (Torres, 2003; Macías y Pérez, 2009). También dio impulso al sector público turístico por la incapacidad y falta de interés del sector privado, destacando la creación en 1974 del Fondo Nacional de Fomento al Turismo que asumió la construcción en Cancún de los hoteles, áreas residenciales, condominios, club de golf, centro de convenciones y el centro urbano para una población de empleados de cuatro mil habitantes, así como el Aeropuerto Internacional. Todo esto creó un flujo de trabajadores hacia el nuevo polo laboral y hacia 1973 la ciudad de servicios tenía seis mil personas en campamentos y asentamientos irregulares, con crecientes problemas de logística por alimentos, energía y sanidad compartidos con la zona turística (Romero, 2009: 20-26, 39, 46; Fernández de Lara, 2009: 167; Macías y Pérez 2009: 244).

\section{Conclusiones}

La historia de las infraestructuras y de los flujos permite tener otra visión sobre la historia de los medios de transporte modernos en América Latina; una primera diferencia reside en considerar las dimensiones fijas, las instalaciones, la materialidad, la cobertura territorial que hacen posible la movilidad. Una segunda diferencia es que el estudio de los trazos, paradas, rutas y tiempos de desplazamiento indican que hasta bien entrado el siglo XX en amplias regiones latinoamericanas los desplazamientos siguieron dándose a través de lentas y largas jornadas. Esto nos conduce a considerar las limitaciones impuestas por la infraestructura de caminos, instalaciones y equipos, así como las rutas y puntos de control establecidos por motivos de orden político como económico, tal como fue la división del trabajo entre el Estado y el capital privado, o bien privilegiar la conexión con una frontera terrestre de una gran economía frente al desplazamiento interno. 


\section{Referencias bibliográficas}

BOGGIO VÁZQUEZ, J. (2008). "Emprendimientos empresariales en el Caribe mexicano: innovación y relaciones entre turismo e infraestructura aeroportuaria a inicios del siglo XXI", en: GUAJARDO, G. (coordinador). Innovación y empresa: estudios históricos de México, España y América Latina. México: Centro de Investigaciones Interdisciplinarias en Ciencias y Humanidades de la Universidad Nacional Autónoma de México-Fundación Gas Natural.

CÁCERES MUÑOZ, J. (2004). "Los obstáculos al crecimiento local: Estado nacional, infraestructura caminera y poder rural en Colchagua durante el siglo XIX", en Mapocho, 55. Santiago: Biblioteca Nacional de Chile, p. 61-70.

CHAPMAN, J. (1975). La construcción del Ferrocarril Mexicano:1837-1880. México: Secretaría de Educación Pública.

EDGERTON, D. (2007). The Shock of the Old: Technology and Global History Since 1900. Oxford: Oxford University Press.

FERNÁNDEZ DE LARA, A. (2009). "Cancún. Las contradicciones socio-ambientales de un Desarrollo Turístico Integralmente Planeado: 1970-2000", en: MACÍAS, C. y PÉREZ, R. (coordinadores). Cancún: los avatares de una marca turística global. México: Bonilla Artigas Editores, Universidad de Quintana Roo, Conacyt.

FERROCARRILES DEL ESTADO (1966). Itinerarios trenes de pasajeros. Red Norte (Calera a lquique y Ramales). En vigencia desde el 16 de octubre de 1966. Para uso exclusivo del personal. Santiago de Chile: Talleres Gráficos de los FF.CC. del Estado.

GAY, C. (1973). Agricultura chilena. Santiago de Chile: ICIRA, Tomo II, 2a. ed.

GUAJARDO, G. (2007). Tecnología, Estado y ferrocarriles en Chile, 1850-1950. Madrid-México: Fundación de los Ferrocarriles Españoles-CEIICH/UNAM.

GUAJARDO, G. (2008). "Cambios tecnológicos y proyectos económicos en las fuerzas armadas de Chile, 1860-1930", en Historia, 41, II. Santiago: Instituto de Historia, Pontificia Universidad Católica de Chile, p. 371-412.

GUAJARDO, G. (2010a). "Mobility History in Modern Mexico: an uneven landscape", en MOM, G.; NORTON, P.; CLARSEN, G. y PIRIE, G. (editores). Mobility in History: Themes in Transport: T2M Yearbook 2011. Neuchâtel, Suisse: Éditions Alphil - Presses Universitaires Suisses.

GUAJARDO, G. (2010b). Trabajo y tecnología en los ferrocarriles de México: una visión histórica, 1850-1950. México: Consejo Nacional para la Cultura y las Artes, Colección El Centauro.

GUAJARDO, G. y RIGUZZI, P. (2013). "Railroad Culture and Mobility in Twentieth Century Mexico", en: Tinajero, A. y Freeman, J. B. (editors). Technology and 
Culture in Twentieth Century Mexico. Tuscaloosa, Alabama: The University of Alabama Press.

LEAL, J. y WOLDENBERG, J. (1986). Del Estado liberal a los inicios de la dictadura porfirista. México: Instituto de Investigaciones Sociales de la Universidad Nacional Autónoma de México- Siglo Veintiuno Editores.

MACÍAS, C. y PÉREZ, R. (coordinadores) (2009). Cancún: los avatares de una marca turística global. México: Bonilla Artigas Editores, Universidad de Quintana Roo, Conacyt.

MARÍN VICUÑA, S. (1914). Explotación del Ferrocarril Longitudinal. Santiago: Imprenta Universitaria.

PRELORENZO, C. (2007). L'immobilité des infrastructures. En: PRELORENZO, C. y ROUILLARD, D. (editores). Le Temps des Infrastructures. París: L'Harmattan.

ROMERO, R. (2009). "Política municipal y desarrollo urbano de un modelo turístico. Cancún: 1975-2002", en: MACÍAS, C. y PÉREZ, R. (coordinadores). Cancún: los avatares de una marca turística global. México: Bonilla Artigas Editores, Universidad de Quintana Roo, Conacyt.

RUIZ, R. (1992). Hombres nuevos: metodismo y modernización en México, 18731930. México: Centro de Comunicación Cultural.

TORRES, R. (2003). "Linkages between tourism and agriculture in Mexico", en Annals of Tourism Research, Vol. 30, 3, p. 546-566.

VAN DER VLEUTEN, E. (2008). "Toward a Transnational History of Technology: meanings, promises, pitfalls", en Technology and Culture, Vol. 49, 4, p. 974-994. VAN HOY, T. (2008). A Social History of Mexico's Railroads: Peons, Prisoners, and Priests. Lanham, Maryland: Rowman\& Littlefield.

WILKIE, J. (1987). La Revolución Mexicana (1910-1976). Gasto federal y cambio social. México: Fondo de Cultura Económica. 